\title{
Lymphoproliferative Erkrankungen der Niere und ableitenden Harnwege
}

M. van den Brand, J. H. J. M. van Krieken

Inhalt

Niere

930

Harnröhre

933

Harnleiter

931

Literatur

935

Harnblase

932 
Niere

Einleitung und Definition. Lymphome in der Niere Eine sekundäre Beteiligung der Niere durch disseminierte Lymphome ist häufig und wurde bei Autopsien bis zur Hälfte der Patienten mit fortgeschrittenen Lymphomen beobachtet $[60,74,90]$. Bei Staging-Verfahr wurde dagegen ein Lymphombefall der Nieren nur in 3-8 \% der Patienten entdeckt [92

Primäre Nierenlymphome sind viel seltener und $\mathrm{m}-$ chen $<1 \%$ der extranodalen Lymphome aus [31]. Lymphome können auch bei der immunsuppressiven $\mathrm{Be}$ handlung nach Organtransplantation auftreten; hierbe sind Nierentransplantationen ein Prädilektionsort fur ymphoproliferative Erkrankungen nach Organtran plantation (PTLD).

Klinik. Patienten mit primärem Nierenlymphom er kranken meist in mittlerem bis hoherem Lebensalters, nur selten wurden Fälle bei Kindern berichtet [81]. In der Regel ist nur eine Niere beteiligt, jedoch treten bilaterale Erkrankungen bei einer kleinen Untergruppe vo Patienten auf und sind häufig mit Niereninsuffizien assoziiert $[6,9,28,30]$.

Klinisch haben die Patienten meist Flankenschmeren, eine Hämaturie sowie Allgemeinsymptome wie Appetitlosigkeit, Übelkeit, Fieber, Gewichtsverlust, un Unpässlichkeit $[21,28,76]$. Ein falsch-positiver Anstieg des prostataspezifischen Antigens (PSA) im Serum wurde bei einem einzigen Patienten berichtet [22].

Obwohl eine sekundäre Nierenbeteiligung bei einem hohen Prozentsatz von Patienten mit disseminierten Lymphomen festgestellt werden kann, zeigen diese Pa tienten in der Regel keine klinischen Symptome einer Niereninfiltration.

Wichtig ist, dass bei der Bildgebung von primären Nierenlymphomen ähnliche Befunde wie bei Nierenzellkarzinomen erhoben werden und die Differenzierung zwischen Karzinom und Lymphom, basierend nur au klinischen Eigenschaften und Bildgebung, sehr schwieri oder unmöglich sein kann $[17,21]$. Allerdings sollte be bilateralem oder multifokalem Befall, bei knotenformmige Infiltration des perirenalen Gewebes und extrarenaler
Tumorausdehnung sowie massiver Lymphadenopathie in erster Linie an ein Nierenlymphom gedacht werden [21].

Morphologie. Makroskopie: Makroskopisch kann der primäre Nierenbefall durch ein Lymphom sich als einzrimarer Tumor oder als multifokale Tumorinfiltratio präsentieren. Die Tumorgröße ist variabel und reich von einem theinen Tumor bis zu einer massiven Infitration der cesamten Niere $[30,68]$ und zum Mithefoll von benarten Geweben und Organen. Der Tum kn sich auch in

wie bei Nierenzellkarzinomen $[21,25,96]$. Auch eine extrahepatische Obstruktion der Gallenwege durch Lymphominfiltration wurde berichtet [1]

Auf der Schnittlache sind Nierenlymphome in der Regel homogene, fleischig bis feste oder harte Tumoren aber auch pseudozystische Veränderungen, Nekrosen und Blutungen können auftreten [28,92].

Histologie: Die primarr in der Niere auftretenden Lymphomentitäten sind sehr variabel. Diffuse großzellige B-ZellLymphome sind der häufigste Subtyp, sowohl von primärem als auch sekundarem Lymphombefall der Nieren ([21, 28, $67,90,101] ;$ Abb. 36.1). Andere Lymphome, die auftreten konnen, sind das extranodale Marginalzonen-B-Zell-Lymphom vom MALT-Typ [33], das kleinzellige lymphozytische Lymphom, das follikuläre Lymphom [28], lymphoplasmazytische Lymphome [28], Burkitt-Lymphom [23, $76,81]$, lymphoblastische Lymphome [11], Plasmozytom $[26,39]$ und reife T-Zell-Lymphome [1].

MALT-Lymphome wurden in der Niere nur selten beobachtet, sowohl als sekundarer Befall bei Vorliegen von MALT-Lymphomen an anderer Stelle als auch als primäLymphom $[16,33,41,52,55,72,73,91]$.

Histologisch ähneln diese Lymphome den MALTLymphomen anderer Lokalisation: Sie bestehen aus keinen lymphozytischen Zellformen mit leicht unregelmäßigen Zellkernen. Typische monozytoide Zellen kommen in einem Teil der Fälle vor; eine Plasmazelldifferenzierung ist häufig [33]. Reaktive Keimzentren sind oft vorhanden. Lymphoepitheliale Läsionen wurden in einigen Fallen gefunden $[16,41,73]$. Eine chromosomale Translokation des MALT1-Gens, wahrscheinlich mit dem Immunglobulin-Schwerkettenlokus, wurde in einem einzigen Fall von primärem MALT-Lymphom der Niere festgestellt [33]

Das intravaskuläre Lymphom neigt zu einer Beteiligung der Nieren $[29,98]$. Diese seltenen Lymphome sind durch eine ausschließliche oder überwiegende Lokalisation der Lymphomzellen in den Gefäßlichtungen charakterisiert. In der Niere werden glomeruläre und peritubuläre Kapillaren durch große, blastäre lymphatische Tumorzellen infiltriert. Obwohl eine Nierenbeteiligung bei der Mehrzahl der Patienten besteht, wird das Krankheitsbild zusätzlich zur B-Symptomatik durch Symptome dominiert, die von der Beteiligung des zentralen Nervensystems und der Haut herrühren. Das intravaskuläre Lymphom hat in der Regel einen B-ZellPhänotyp und entspricht so dem intravaskulären diffusen großzelligen B-Zell-Lymphom, selten besteht ein T-ZellPhänotyp eines anaplastisch-großzelligen Lymphoms $[20,35,87]$. Wegen der häufigen Nierenbeteiliging des intravaskularen Lymphoms wurde die Nierenbiopsie als diagnostische Maßnahme vorgeschlagen, um bei klinischem Verdacht Gahme vorgeschlagen, um bei kinischem Verdacht Gewebe zur Diagnosesicherung und
Therapieevaluation zu entnehmen $[2,5]$.
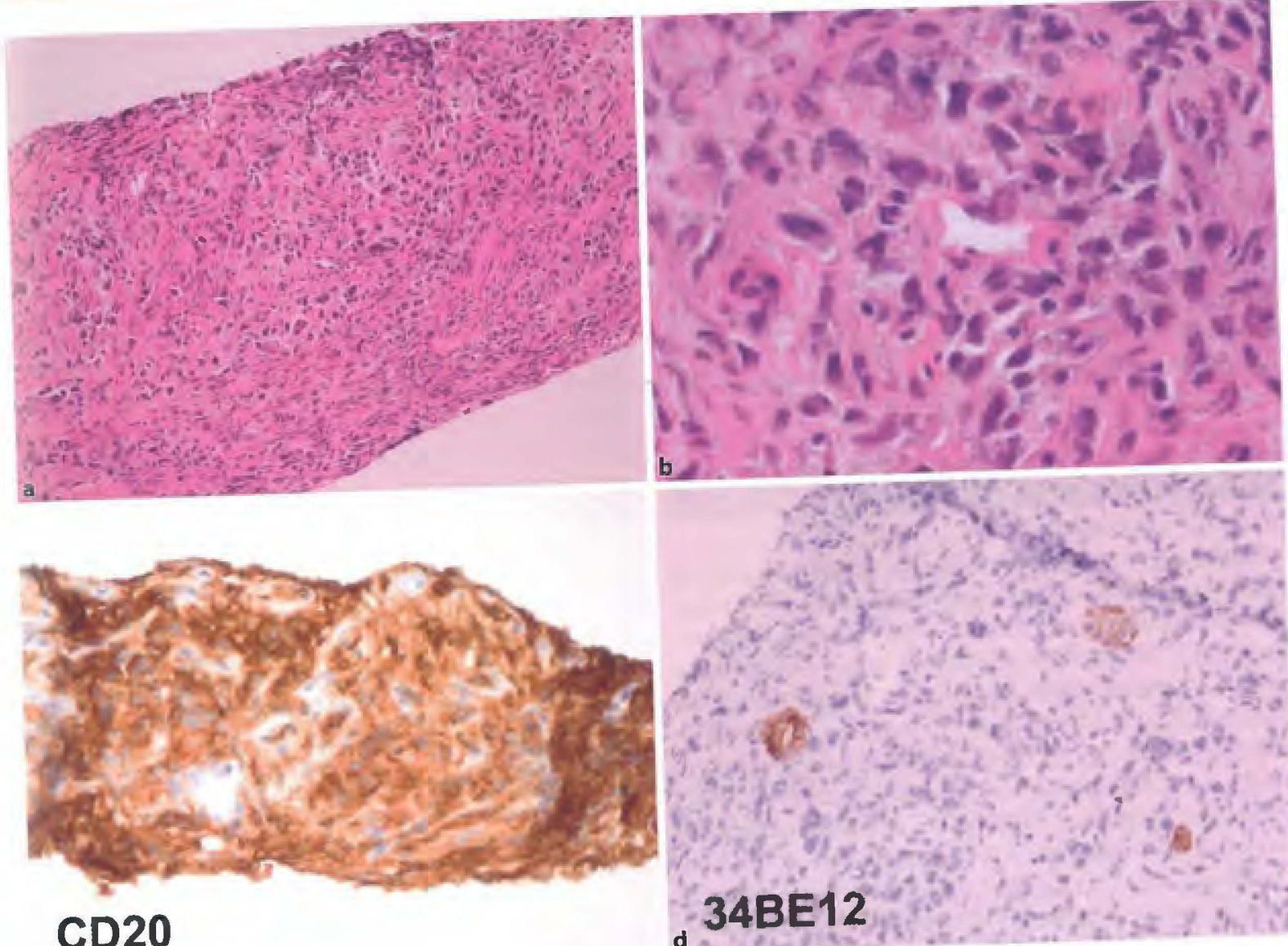

CD20

Abb. 36.1 a-d Stanzbiopsat der Niere mit Befall durch ein diffuses Abb. grobzelliges B-Zell-Lymp as. Bei höherer Vergrößerung (b) aus gro-

Ben atypischen Blasten besteht. $c$ Das Inflas weis von $\mathrm{CD} 20$. d Zytokesti

phoproliferative Erkrankungen nach Organtransplantation befallen [15].

Die Mehrheit der Lymphoproliferationen in diesen Fällen sind EBV-assoziiert und reichen von einer polyklonalen lymphatischen Hyperplasie bis zu monoklonalen Neoplasien, die von herkömmlichen Hodgkin- oder Non-Hodgkin-Lymphomen nicht $\mathrm{zu}$ unterscheiden sind. Lymphome, die bei Nierentransplantation entstehen, sind meist auf den Translantatempfanger zurte zuführen, jedoch wurden auch Lymphome berichtet, die ihren Ausgang von Spenderlymphozyten nehmen $34,50,59$

Harnleiter

direkte Infiltration aus dem Retroperitoneum oder ausrehend von den Nieren oder dem Genitaltrakt ist die gehonds Ursache der urothelialen Beteiligung $[30,53]$. Das primäre urethrale Lymphom ist sehr selten und die Diteratur beschränkt sich auf Fallberichte.

linik. Die Lymphominfiltration der Harnleiter wird fast immer von einer Hydronephrose oder von Hydrou enter $[9,48,49,50,51]$. Patienten konnen symptomatisch sein, aber auch mit Flanken- oder Hückenshmerzen einhergehen. Eine Hämaturie kann ebenfalls bestehen [64]

Morphologie. Primäre Lymphome des Harnleiters sin auf Fallberichte mit lerweise sind diese Lymphome unlateral, jedoch wurde eine beidseitige urethrale Beteilateral, jedoch wurde eine beidseitige $[51,53]$.

Einführung. Ein Lymphombefall des Harleiters trit le Fine Pränlenz für bestimmte Abschnitte der Harnmeist bei disseminierten Lymphomen auf. In einer Au- Eine Pravalenz fur bestewiesen werden $[45,51,64]$

topsieserie von 127 Patienten mit Non-Hodgkin-Lym- leiter konnte nicht nachgewesen-Zell-Lymphomen [17] phom zeigten $16 \%$ eine Harnleiterbeteiligung [77]. Ein 
kommen follikuläre Lymphome [45, 51, 64], kleinzellig Harnleiterobstruktion und Hydronephrose durch Tulymphozytische Lymphome [53] und MALT-Lymphome $[36,70]$ vor.

Differenzialdiagnose. Differenzialdiagnostisch muss das Lymphoepitheliom-artige Karzinom abgegrenz werden, das in Harnleiter und Nierenbecken vereinzelt beobachtet wurde $[36,70]$. Diese Tumoren bestehen aus undifferenzierten Tumorzellkomplexen mit einem prominenten reaktiven lymphatischen Infiltrat. Der immunhistochemische Nachweis von Zytokeratinen beweist den epithelialen Ursprung dieser Tumoren [86]

\section{Harnblase}

Einführung. Lymphome der Harnblase sind selten. Sie betreffen weniger als $1 \%$ aller Blasentumoren [7]. Ein Lymphombefall der Harnblase wird als primär angesehen, wenn eine Beteiligung benachbarter Gewebe oder anderer Organe fehlt. Disseminierte Lymphome, die zuerst durch den Befall der Harnblase diagnostiziert werden, werden als Lymphome ohne Primärlokalisation bezeichnet. Ein sekundärer Lymphombefall liegt vor, wenn ein Lymphom einer anderen Primärlokalisation auch die Blase beteiligt.

Bei gezielter Suche ist ein sekundärer Lymphombefall der Harnblase durchaus nicht selten und tritt in $\mathrm{Au}$ topsieberichten bei 10-20\% der Patienten auf in AuBlasenlymphome sind dagegen viel seltener, sie betreffen weniger als $1 \%$ der extranod vel seltener, sie betreffe weniger als $1 \%$ der extranodalen Lymphome [31]. In Lymphome der Blase gelegt.

Klinik. Primäre Harnblasenlymphome betreffen vor作 twa 70 Jan wilit 2 ischen der driten bis neun Dekade [38]. . Clinis Whing ein lamature, Vorgeschichte Blasenentzündungen $[38,65,82]$. Selten ist in der Vorgeschichte eine interstitielle Zystitis be primären Blasenlymphomen

Morphologie. Makroskopisch ist der Tumor bei etwa wei Drittel der Patienten solitär sowie sessil und ähnelt den Urothelkarzinomen $[7,85]$. Multiple sessile Tumoen, polypöse Tumoren oder eine diffuse Infiltration der [7, 46] Auch die Seitenwän ten $[7,46]$. Auch die Seitenwande, u. U. mit mehreren Tumoren, und das Trigonum der Blase kônnen bete Lokalisationen variiert von Studie zu Studie $[46,85]$. morobstruktion treten bei hochmalignen B-Zell-Lymphomen am häufigsten auf $[46,64,65,85]$, aber auch in sehr selten Fällen bei niedrigmalignen Lymphome

Histologie: Das extranodale Marginalzonenlymphom vom MALT-Typ (MALT-Lymphom) ist das häufigste primäre Lymphom der Harnblase. Histologisch ähnelt es den in anderen extranodalen Lokalisationen auftretenden MALT-Lymphomen. Es besteht aus kleinen lymschen Zellen reichen. Eine Plasmazelldifferenzierung is häufig und größere Blasten sind locker eingestreut. Die Blasenwand wird diffus infiltriert; auch die Schleimhaut und die darunterliegende Muskulatur sind dabei beteiligt [46]. Lymphoepitheliale Läsionen sind oft vorhanden und können sich in Verbindung mit einer Cystitis den und können sich in Verbindung mit einer Cystitis cystica, einer Cystitis glandularis $[3,46,71]$ oder im
Ubergansepithel ausbilden [7]. Reaktive Keimzentren sind ebenfalls häufig. Eine Kolonisierung durch die neohistochemische Profil entspricht dem der MALT-Lymphome mit Expression von Pan-B-Zell-Markern (CD20 Keimzentrumsmarkern. Auch andere niedrigmalign B-Zell-Lymphome können primär in der Harnblase auftreten (Abb. 36.2). das zweithäufigste, primäre Lymphom der Harnblase. lisation, mit einer diffusen Infiltration durch große, bastäre B-Zellen (Abb. 36.3). Das DIBCL kann de novo in der Blase entstehen, jedoch wurde auch die Transformation eines niedrigmalignen B-Zell-Lymphome mitgeteilt $[7,100]$

Andere B-Zell-Lymphome, die nur sehr selten primär in der Blase auftreten, sind u. a. das follikuläre Lymphom [78], das Burkitt-Lymphom [58] und das plasma([88]; Abb. 36.4).

Periphere T-Zell-Lymphome der Harnblase sind seh selten und stellen meist eine sekundäre Organbeteiligung dar Primäre T-Zell-Iymphome wurden nur verinzelt berichtet $[14,38,62]$, wobei bei einem Fall Schistosomiasis bestand [62]

Hodgkin-Lymphome der Harnblase sind sehr selten und treten in der Regel sekundir au Lymphom anderer Pinger Todgkin-Lymphom der Harnbse ist noch wesentich . [42].

Differenzialdiagnose. Harnblasenkarzinome können auch histologisch einem Blasenlymphom ähneln [24]. So kann eine sehr prominente Lymphozyteninfiltration das darunterliegende Karzinom verdecken [102]. Auch kann phatischen Zellen, die von monozytoiden zu zentrozytiplastischen Zellen kann bestehen [56,63]. Das immunCD79a), jedoch einem Fehlen von CD5, Cyclin D1 ode

Das diffuse, großzellige B-Zell-Lymphom (DLBCL) ist Histologisch entspricht es den DLBCL anderer Loka

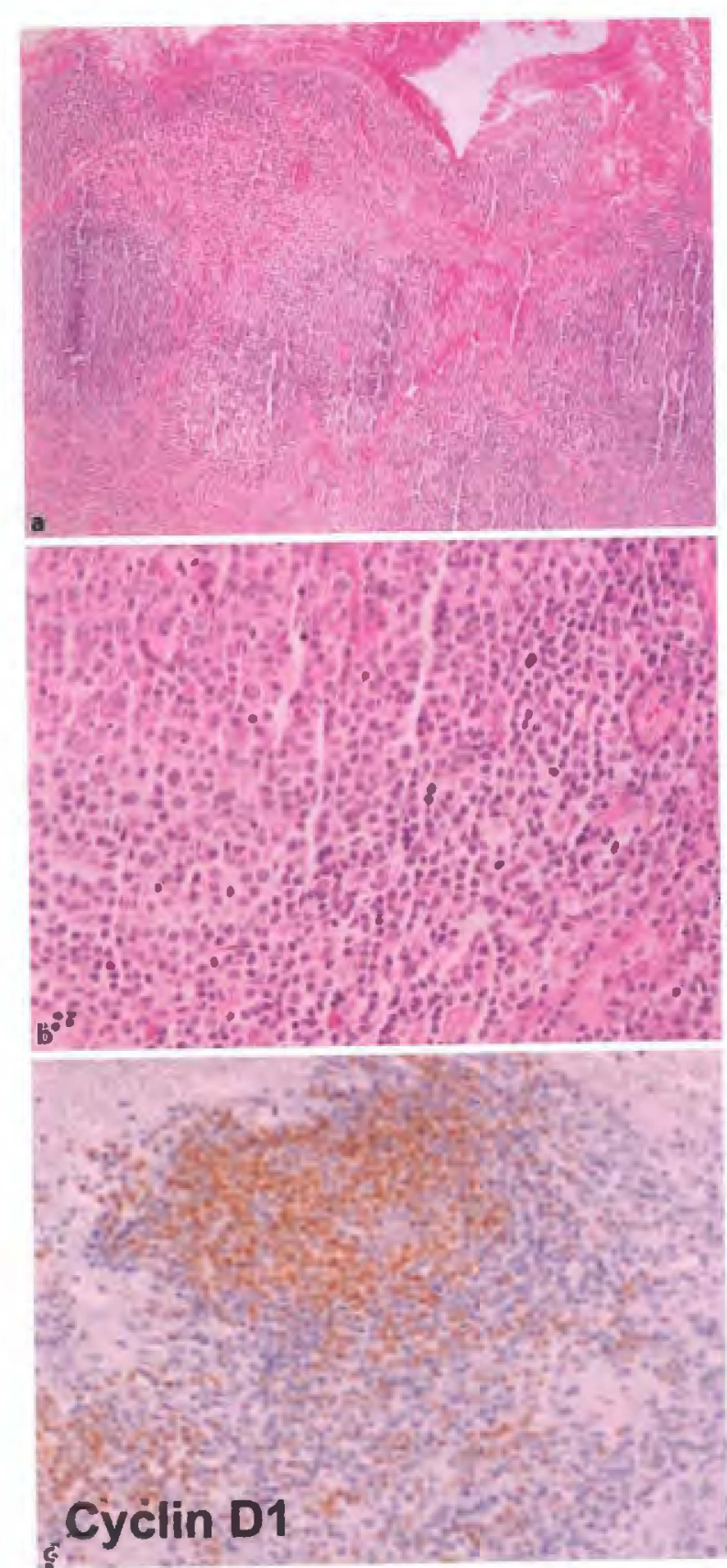

Abb. 36.2 ac Mantelzelliymphom in sity der Harnblase Die Schleimh a tist $z$ m chleim a $c$ ist 2 The

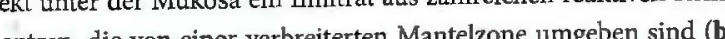
Der in positive Reaktion in einem Teil der Lymphozyten, passend zur Diagnose eines Mantelzellyymphoms. Durch Fluoreszenz-in-situPage Hybridisie
Der Nachweis von höher differenzierten Karzinomanteilen oder das Vorhandensein einer Urotheldysplasie bzw. eines Carcinoma in situ sprechen für das Vorliegen eines Karzinoms. Die Ims epitheliale Natur des Tum weisen. Auch solte man die Diagnose eines Lymphoms Markern nachgewiesen werden kann.

Die Unterscheidung zwischen einer ausgeprägten chronischen Entzündung der Harnblase und einem niedrigmalignen Lymphom kann sehr schwierig sein [24, 71]. Zu den Merkmalen, die für die Diagnose eines Lymphoms sprechen, zählen das Vorliegen eines monomorphen Infiltrats und das Vorhandensein von lymphoepithelialen Läsionen. Weiterhin können Immunhistochemie und Untersuchungen der Klonalität helfen Die inflammatorischen myofibroblastischen Tumoren (IMT) der Harnblase bestehen aus Wucherungen vo Spindelzellen, die das ALK-1-Protein exprimieren kônnen. In seltenen Fallen kann es deshalb schwierig sein, den IMT von der sehr seltenen sarkomatoiden Variante des anaplastischen, großzelligen Lymphoms zu unterscheiden, das ebenfalls Alk-positiv ist und dem IM morphologisch ahneln kann [4]. Neben den morphologischen Eigenschaften, können immunhistochemische diese Differenzialdiagnose zu klären [4]

Therapie und Prognose. Patienten mit primärem niedrigmalignen B-Zell-Lymphom der Harnblase wurde entweder durch operative Entfernung, Strahlentherapie Chemotherapie oder mit einer Kombination dieser Therapien behandelt [38]. Der Erfolg dieser Behandlungen war bei den meisten Patienten ahnlich und fuhrte $\mathrm{zu}$ vollständiger Rückbildung [38]. Aufgrund der hohen Erfolgsrate von Chemotherapie und Strahlentherapie, scheint für die chirurgische Behandlung nur wenig Spielraum zu bestehen [38]. Bei der Behandlung von hochmalignen Lymphomen wird die Chemotherapie derzeit bevorzugt, weil diese Therapie auch bei einer nicht nachweisbaren, frühen systemischen Ausbreitung wirksam ist [38].

Bei der Assoziation von chronischer Zystitis und MALT-Lymphom stellt sich die Frage, ob Infektionen eine Rolle bei der Entwicklung des Lymphoms spielen, ähnlich wie bei MALT- Lymphomen in anderen Organen. Tatsächlich konnte nach Antibiotika-Therapie eine komplette Rückbildung in Fällen des primären MALTLymphoms der Harnblase erzielt werden $[32,38,69,93]$.

Harnröhre

Einleitung. Die Harnröhre ist der ungewöhnlichste Or für ein primär entstandenes Non-Hodgkin-Lymphom 

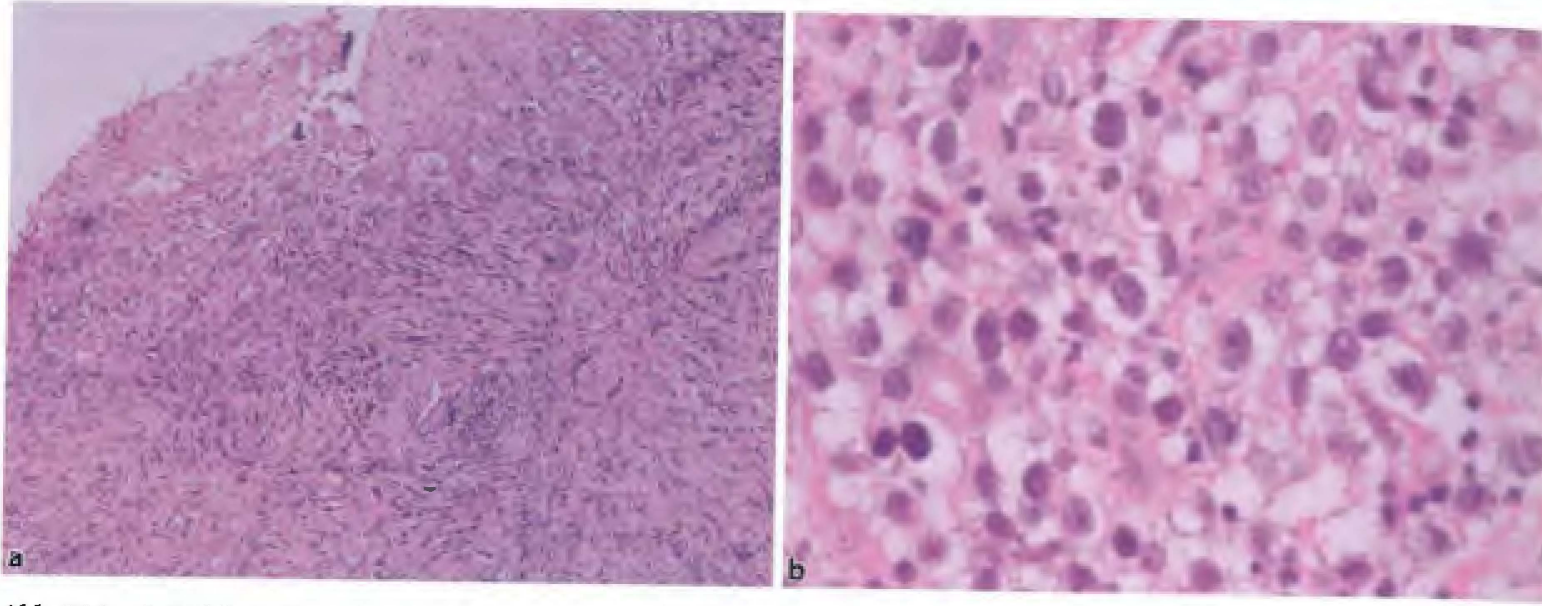

Abb. 36.3 a,b Befall der Harnblase durch ein diffuses großzellige B-Zell-Lymphom. a Bei niedriger Vergrößerung ist die Mukosa teilweise erhalten. $\mathbf{b}$ Im darunter gelegenen Gewebe besteht eine Infil-

\section{tration durch große, atypische Zellen. Immunhistochemisch war der} DLBCL

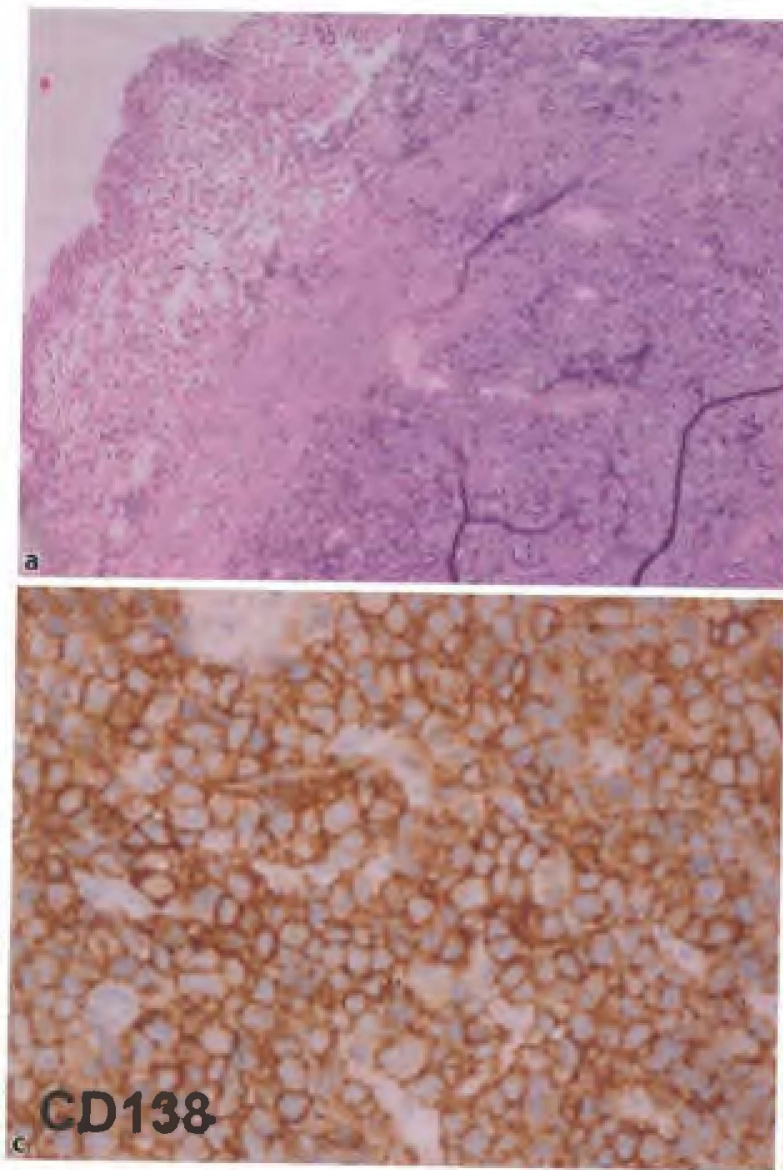

Abb. 36.4 a-d Plasmoblastisches Lymphom der Hornobse einem HIV-positiven Patienten. a Bei kleiner Vergrößerung ist die
Schleimhaut und Submukosa intakt. b Unter der Submukosa besteht ein dichtes Infiltrat aus Zellen mit großen, unregelmäßigen Zell-

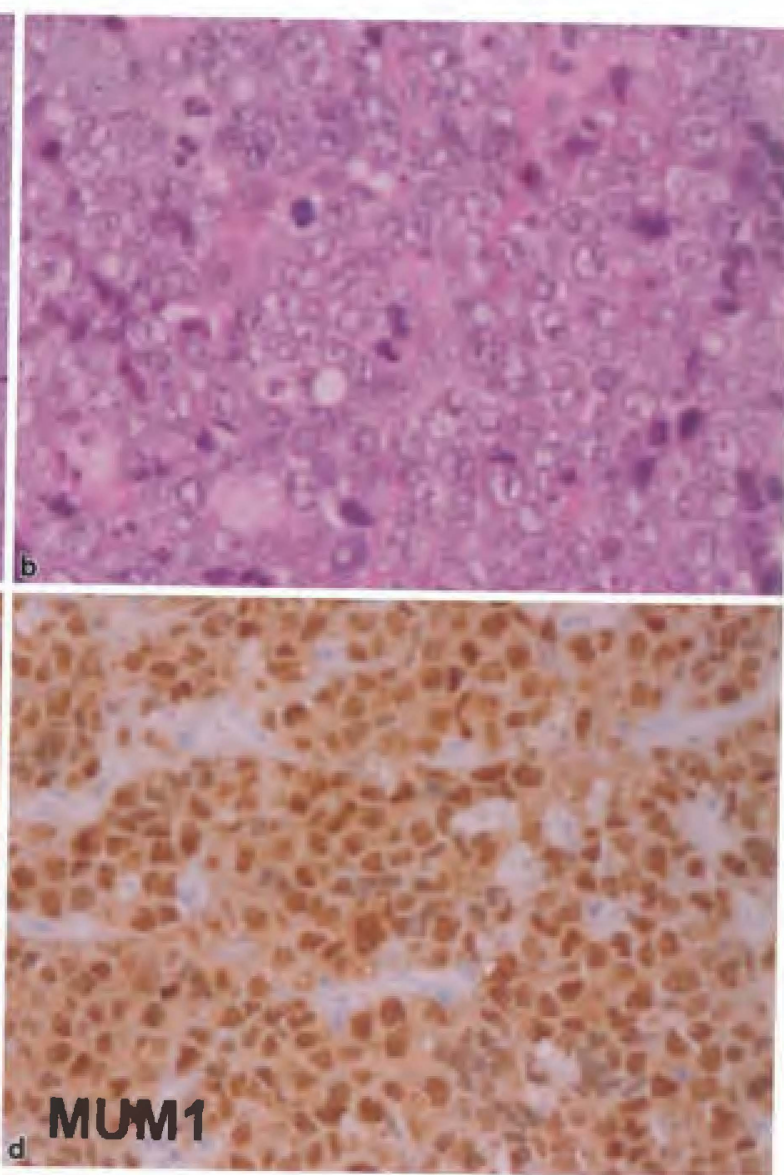

kernen. Diese Zellen waren negativ beim Nachweis von CD20 (nicht gebildet), zeigten aber eine starke Expression von CD138 (c) und phoms im Harntrakt. Die Literatur über primäre Lymphome der Harnröhre beschränkt sich auf Fallberichte $m$ weniger als 20 berichteten Fällen. Auch eine sekundäre Beteiligung der Harnröhre durch ein Lymphom ist selten [30].

Klinik. Frauen scheinen häufiger betroffen zu sein als Männer. Die meisten Patienten erkranken im mittleren bis hohen Alter; es gibt aber auch Berichte von Patienten mit primärem Harnröhrenlymphom in jüngerem $E_{r-}$ krankungsalter. Bei Frauen waren die führenden Krankheitssymptome Hämaturie, Dysurie, Polyurie, duplizierter Harnstrahl, vaginale Schmierblutungen, das Gefüh von Restharn, Bauchschmerzen und das Vorhandensein eines polypösen Tumors $[19,40,43,47,56,63,66,79$ $80,82,89,94,95]$. Bei einigen Patienten bestand eine Harnröhrenkarunkel [43, 47, 95]. Bei Männern waren die Leitsymptome Hämaturie, Blasenentleerungsstörungen und verzögerter Harnfluss [37, 48, 54, 57, 75]. Harnwegsinfektionen, Arthritis, HIV und benigne Prostatahyperplasie wurden in Assoziation mit einem primären Harnröhrenlymphom berichtet [37, 54, 82, 89].

Morphologie. Makroskopisch besteht in den meisten Fällen ein polypöser Tumor, aber es gibt auch Fälle, be denen die Harnröhre zirkulär und auch die benachbarten Organe infiltriert sind $[30,57,82,89]$. Am häufigsten kommen MALT-Lymphome vor; diese scheinen eine günstigere Prognose als andere Lymphome der eine günstigere Prognose als andere Lymphome der
Harnröhre zu haben [57]. Andere B-Zell-Lymphome, Harnröre zu haben [57]. Andere B-Zell-Lymphome, großzellige B-Zell-Lymphom [37, 66, 75, 80, 82, 94, 95] und das follikulare Lympom [48]. T-Zell-Lymphom wurden nur sehr selten in der Harnröhre berichtet.

\section{Literatur}

1. Abbas Z Johnston DA, Murray FE (1996) Renal lymphoma: an unusual cause of extrahepatic biliary obstruction Postgrad Med J72.617-618

2. Agar JW, Gates PC Vaughan SL Machet (1994) Rena biopsy in angiotropic larg cell lymphom Am J Kidney Dis 24:92-96

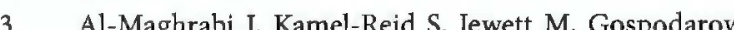

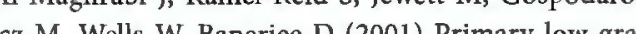
B-cell lymphoma of mucosa-asocis sue type arising in the urinary bladder: rymphoid tiswh malch with molecular genetic analysis. Arch Pathol Lab Med

4. Allory Y, Merabet Z, Copie-Bergman C, Lange F, Yiou R, Gaulard P (2005) Sarcomatoid variant of anaplastic are cellympos mimics ALK-1-positive inflic ry my cell ly ne 29:838-839 (author reply 839 )
Axelsen RA, Laird PP, Horn M (1991) Intravascular large cell lymphoma: diagnosis on renal biopsy. Pathoogy 23:241-243

Baldus M, Klooker P, Kress S, Waldherr R, Moller P, Brass $H$ (1996) Primary bilateral renal centrocytic nonHodgkin's lymphoma as a cause of renal failure. Nephron 73:86-90

Bates AW, Norton AJ, Baithun SI (2000) Malignant lymphoma of the urinary bladder: a clinicopathological study of 11 cases. J Clin Pathol 53:458-46

8. Bocian JJ, Flam MS, Mendoza CA (1982) Hodgkin's disease involving the urinary bladder diagnosed by urinary cytology: a case report. Cancer 50:2482-2485

Brouland JP, Meeus F, Rossert J, Hernigou A, Gentric D, Jacquot C, Diebold J, Nochy D (1994) Primary bilatera B-cell renal lymphoma: a case report and review of the literature. Am J Kidney Dis 24:586-589

10. Buck DS, Peterson MS, Borochovitz D, Bloom EJ (1992) Non-Hodgkin lymphoma of the ureter: CT demonstration with pathologic correlation. Urol Radiol 14:183-187

11. Camitta BM, Casper JT, Kun LE, Lauer SJ, Starshak R, Oechler HW (1986) Isolated bilateral T-cell renal lymphoblastic lymphoma. Am J Pediatr Hematol Oncol 8:8-12

12. Chen HH, Panella JS, Rochester D, Ignatoff JM, McVary KT (1988) Non-Hodgkin lymphoma of ureteral wall: CT findings. J Comput Assist Tomogr 12:157-158

13. Cheung AN, Chan AC, Chung LP, Chan TM, Cheng IK, Chan KW (1998) Post-transplantation lymphoproliferative disorder of donor origin in a sex-mismatched renal allograft as proven by chromosome in situ hybridization. Mod Pathol 11:99-102

14. Choi JH, Jeong YY, Shin SS, Lim HS, Kang HK (2003) Primary calcified T-cell lymphoma of the uring

15. Cohen JI (1991) Epstein-Barr virus lymphoproliferative disease associated with acquired immunodeficiency Medicine (Baltimore) 70:137-160

6. Colovic M, Hadzi-Djokic I Cemerikic V Colovic R, ankovic C Dacic M (1999) Primary MALT lympon, of the kidney. Hematol Cell Ther 41:229-232

17. Curry NS, Chung CJ Potts W, Bissada N (1993) Isolated (1993) Isolated 41:494-498

18. de Leval L, Jardon-Jeghers C, Gennigens C, Boniver (2006) Hodgkin's lymphoma presenting as a bladder tumour. Haematologica 91:ECR3

19. Dell'Atti C, Missere M, Restaino G, Carlino S, Cucci F the female urethra. Rays 30:269-272

DiGiuseppe JA, Nelson WG, Seifter EJ, Boitnott JK, Mann RB (1994) Intravascular lymphomatosis: a clinicopathologic study of 10 cases and assessment of response to chemotherapy. J Clin Oncol 12:2573-2579 
21. Dimopoulos MA, Moulopoulos LA, Costantinides C, 36. Hara M, Satake M, Ogino H, Itoh M, Miyagawa H,
Deliveliotis C, Pantazopoulos D, Dimopoulos C (1996) Deliveliotis C, Pantazopoulos D, Dimopoulos C (1996) Primary renal lymphoma: a clinical and radiological

Djavan B, Keffer JH, Molberg K, Roehrborn CG (1995) False-positive serum prostate-specific antigen values in a patient with non-Hodgkin lymphoma of the kidne

Donadieu J, Patte C, Kalifa C, Lemerle J (1992) Diagno tic and therapeutic problems posed by malignant no Hodgkin lymphoma of renal origin in children. Apropos of 7 cases. Arch Fr Pediatr 49:699-704

24. Evans AJ, Dickson BC (2013) Uncommon tumours in the adult bladder: rare entities with recognized diagnostic pitfalls. Diagnostic Histopathol 19:387-397

5. Eyre RC, Huberman MS, Balogh K (1993) Non-Hodgkin's lymphoma of the kidney with inferior vena caval

entension. Urol Int 51:43-45

6. Fan F, Deauna-Limayo D, Brantley Thrasher J, Dam janov I (2005) Anaplastic plasmacytoma of the kidney.

Histopathology 47:432-433

. Farr MJ, Newling DW, Raper C (1978) Simple chemotherapy for bladder lymphoma with bilateral hydronephrosis. Br J Urol 50:286

28. Ferry JA, Harris NL, Papanicolaou N, Young RH (1995) Lymphoma of the kidney. A report of 11 cases. Am Surg Pathol 19:134-144

2. Ferry JA, Harris NL, Picker LJ, Weinberg DS, Rosales ŖK, Tapia J, Richardson EP Jr. (1988) Intravascular lymphomatosis (malignant angioendotheliomatosis). A Bcell neoplasm expressing surface homing receptors. Mod Pathol 1:444-452

30. Ferry JA, Young RH (1997) Malignant lymphoma of the genitourinary tract. Curr Diagn Pathol 4:145-169

31. Freeman C, Berg JW, Cutler SJ (1972) Occurrence and prognosis of extranodal lymphomas. Cancer 29:252-260

Fujimura M, Chin K, Sekita N, Kajimoto S, Kamijima S, Suzuki H, Ichikawa T, Mikami K (2008) Regression of mucosa-associated lymphoid tissue lymphoma of the bladder after antibiotic therapy: a case report. Hinyokika Kiyo 54:783-786

33. Garcia M, Konoplev S, Morosan C, Abruzzo LV, BuesoRamos CE, Medeiros LJ (2007) MALT lymphoma involving the kidney: a report of 10 cases and review of the literature. Am J Clin Pathol 128:464-473

34. Gassel AM, Westphal E, Hansmann ML, Leimenstoll G, Gassel HJ (1991) Malignant lymphoma of donor origin after renal transplantation: a case report. Hum Pathol 22:1291-1293

35. Ghorbani RP, Shokouh-Amiri H, Gaber LW (1996) In tragraft angiotropic large-cell lymphoma of T cell-type in a long-term renal allograft recipient. Mod Pathol 9:671-676 Hashimoto $\mathrm{Y}$, Okabe $\mathrm{M}$, Inagaki $\mathrm{H}(2002)$ Primary ureteral mucosa-associated lymphoid tissue (MALT) lymphoma - pathological and radiological findings. Radiat Med 20:41-44

Hatcher PA, Wilson DD (1997) Primary lymphoma of the male urethra. Urology 49:142-144

. Hughes M, Morrison A, Jackson R (2005) Primary bladder lymphoma: management and outcome of $12 \mathrm{pa}-$ tients with a review of the literature. Leuk Lymphoma 46:873-877

39. Igel TC, Engen DE, Banks PM, Keeney GL (1991) Renal plasmacytoma: Mayo Clinic experience and review of the literature. Urology 37:385-389

40. Inuzuka S, Koga S, Imanishi D, Matsuo T, Kanetake H (2003) Primary malignant lymphoma of the female urethra. Anticancer Res 23:2925-2927

41. Jindal B, Sharma SC, Das A, Banerjee AK (2001) Indoent behaviour of low-grade $B$ cell lymphoma of mucosaassociated lymphoid tissue arising in the kidney. Urol Int 67:91-93

2. Jones MW (1989) Primary Hodgkin's disease of the urinary bladder. Br J Urol 63:438

43. Kakizaki H, Nakada T, Sugano O, Kato H, Yamakawa M (1994) Malignant lymphoma in the female urethra. Int Urol 1:281-282

4. Kashi SH, Murphy JK, Britton JP, Whelan P (1990) Primary lymphoma of the bladder: a clinicopathologica study of 3 cases. Eur Urol 17:186-188

5. Kawashima A, Shiotsuka Y, Nin M, Kokado Y (2005) Malignant lymphoma of the ureter: a case report Hinyokika Kiyo 51:269-272

6. Kempton CL, Kurtin PJ, Inwards DJ, Wollan P, Bostwick DG (1997) Malignant lymphoma of the bladde evidence from 36 cases that low-grade lymphoma of the MALT-type is the most common primary bladder lymphoma. Am J Surg Pathol 21:1324-1333

4. Khatib RA, Khalil AM, Tawil AN, Shamseddine AI Kaspar HG, Suidan FJ (1993) Non-Hodgkin's lymphoma presenting as a urethral caruncle. Gynecol Oncol 50:389-393

. Kitamura H, Umehara T, Miyake M, Shimizu T, Kohda $K$, Ando M (1996) Non-Hodgkin's lymphoma arising in the urethra of a man. J Urol 156:175-176

4. Koike H, Morita T, Tamura Y (2004) Primary malignan lymphoma of the urinary bladder: a case report. Nihon Hinyokika Gakkai Zasshi 95:675-678

50. Konigsrainer A, Steurer W, Schumer J, Geissler D, Mourad M, Squifflet JP, Margreiter R (1993) Transmission of non-Hodgkin's lymphoma through -disastrous result of flbe diagosis ond ind agraftsformation. Transplant Proc 25:3075-3076

51. Kubota Y, Kawai A, Tsuchiya T, Kozima K, Yokoi S, Deguchi T (2007) Bilateral primary malignat lym
52. Laven BA, Orvieto MA, Rapp DE, Shalhav AL, Ozer O Taxy JB, Sokoloff M (2004) Malignant B-cell lymphom in renal cyst wall. Urology 64:590

53. Lebowitz JA, Rofsky NM, Weinreb JC, Friedmann P (1995) Ureteral lymphoma: MRI demonstration. Abdon Imaging 20:173-175

54. Lopez AE, Latiff GA, Ciancio G, Antun R (1993) Lymphoma of urethra in patient with acquired immune deficiency syndrome. Urology 42:596-598

55. Mak SK, Wong PN, Lo KY, Wong AK (1998) Successfu treatment of IgA nephropathy in association with lowgrade B-cell lymphoma of the mucosa-associated lymphoid tissue type. Am J Kidney Dis 31:713-718

56. Mark JA, Pais VM, Chong FK (1990) Plasmacytoma of the urethra treated with transurethral resection and radiotherapy. J Urol 143:1010-1011

57. Masuda A, Tsujii T, Kojima M, Sakamoto S, Moriguch H, Honda M, Yoshida K (2002) Primary mucosa-associated lymphoid tissue (MALT) lymphoma arising from the male urethra. A case report and review of the literature. Pathol Res Pract 198:571-575

58. Mearini E, Zucchi A, Costantini E, Fornetti P, Tiacci E, Mearini L (2002) Primary Burkitt's lymphoma of bladder in patient with AIDS. J Urol 167:1397-1398

59. Meduri G, Fromentin L, Vieillefond A, Fries D (1991) Donor-related non-Hodgkin's lymphoma in a renal allograft recipient. Transplant Proc 23:2649

60. Miyake O, Namiki M, Sonoda T, Kitamura H (1987) Secondary involvement of genitourinary organs in malignant lymphoma. Urol Int 42:360-362

61. Morita K, Nakamura F, Nannya Y, Nomiya A, Arai Ichikawa M, Maeda D, Homma Y, Kurokawa M (2012) Primary MALT lymphoma of the urinary bladder in the background of interstitial cystitis. Ann Hemato 91:1505-1506

62. Mourad WA, Khalil S, Radwi A, Peracha A, Ezzat A 1998) Primary T-cell lymphoma of the urinary bladder. Am J Surg Pathol 22:373-377

63. Nabholtz JM, Friedman S, Tremeaux IC, Cuisenier J, Mansoni H, Douvier S, Arnalsteen C, Collin F, Guerrin $J$ (1989) Non-Hodgkin's lymphoma of the urethra: a rare extranodal entity. Gynecol Oncol 35:110-111

64. Ni BW, Zhong L, Wang T, Chen FY (2014) Malignant ymphoma of the ureter: a case report and literature review. Exp Ther Med 7:1521-1524

65. Ohsawa M, Aozasa K, Horiuchi K, Kanamaru A (1993) Malignant lymphoma of bladder. Report of three cases and review of the literature. Cancer 72:1969-1974

6. Ohsawa M, Mishima K, Suzuki A, Hagino K, Doi Aozasa K (1994) Malignant lymphoma of the urethre: report of a case with detection of Epstein-Barr virus genome in the tumour cells. Histopathelogy 24:525-529

67. Okuno SH Hoyer ID, Ristow $K$, Witzig TE (1995) Pri-

din tranodal site. Cancer 75:2258-2261
68. Osborne BM, Brenner M, Weitzner S, Butler JJ (1987) Malignant lymphoma presenting as a renal mass: fou cases. Am J Surg Pathol 11:375-382

69. Oscier D, Bramble J, Hodges E, Wright D (2002) Regression of mucosa-associated lymphoid tissue lymphoma of the bladder after antibiotic therapy. J Clin Oncol 20:882 Otsuki H, Ito K, Sato K, Kosaka T, Shimazaki H, Kaji T, Asano T (2013) Malignant lymphoma of mucosa-associated lymphoid tissue involving the renal pelvis and the entire ureter: a case report. Oncol Lett 5:1625-1628

. Pawade J, Banerjee SS, Harris M, Isaacson P, Wright D (1993) Lymphomas of mucosa-associated lymphoid tissue arising in the urinary bladder. Histopathology 23:147-151

Pelstring RJ, Essell JH, Kurtin PJ, Cohen AR, Banks PM (1991) Diversity of organ site involvement among malignant lymphomas of mucosa-associated tissues. Am Clin Pathol 96:738-745

Qiu L, Unger PD, Dillon RW, Strauchen JA (2006) Lowrade mucosa-associated lymphoid tissue lymphoma involving the kidney: report of 3 cases and review of the literature. Arch Pathol Lab Med 130:86-89

4. Richmond J, Sherman RS, Diamond HD, Craver LF (1962) Renal lesions associated with malignant lymphomas. Am J Med 32:184-207

7. Richter LA, Hegde P, Taylor JA 3rd (2007) Primary nonHodgkin's B-cell lymphoma of the male urethra presening as stricture disease. Urology 70(1008):e1011-e1002

76. Salem Y, Pagliaro LC, Manyak MJ (1993) Primary small noncleaved cell lymphoma of kidney. Urology 42:331-335

Scharifker D, Chalasani A (1978) Ureteral involvement by malignant lymphoma. Ten years' experience. Arch Pathol Lab Med 102:541-542

78. Schniederjan SD, Osunkoya AO (2009) Lymphoid neoplasms of the urinary tract and male genital organs: a clinicopathological study of 40 cases. Mod Patho 22:1057-1065

Selch MT, Mark RJ, Fu YS, Belldegrun A, Parker RG (1993) Primary lymphoma of female urethra: long-term control by radiation therapy. Urology 42:343-346

80. Shimizu Y, Ogawa O, Terachi T, Okada Y, Yoshida O (1997) A case of primary urethral lymphoma presenting as a huge mass surrounding the female urethra. Hinyokika Kiyo 43:229-232

1. Sieniawska M, Bialasik D, Jedrzejowski A, Sopylo B, Maldyk J (1997) Bilateral primary renal Burkitt lymphoma in a child presenting with acute renal failure. Nephrol Dial Transplant 12:1490-1492

82. Simpson RH, Bridger IE, Anthony PP, James KA, Jury I (1990) Malignant lymphoma of the lower urinary tract. A clinicopathological study with review of the literature. Br J Urol 65:254-260

83. Sosna J, Lossos IS, Libson E (2000) Hodgkin's lymphoma of the urinary bladder. Clin Radiol 55:405-406 
84. Sufrin G, Keogh B, Moore RH, Murphy GP (1977) Secondary involvement of the bladder in malignant lymphoma. J Urol 118:251-253

85. Tasu JP, Geffroy D, Rocher L, Eschwege P, Strohl D, Benoit G, Paradis V, Blery M (2000) Primary malignant lymphoma of the urinary bladder: report of three cases and review of the literature. Eur Radiol 10:1261-1264

86. Terai A, Terada N, Ichioka K, Matsui Y, Yoshimura K, Wani X (2005) Lymphoepithelioma-like carcinoma of the ureter. Urology 66:1109

87. Thiel R, Sarbia M, Gerharz CD, Ebert T, Schmitz-Drager B, Ackermann R (1995) Angiocentric lymphoma of the kidney in the acquired immunodeficiency syndrome. J Urol 153:137-139

88. Tille JC, Pelte MF, Schwartz J, Dietrich PY, McKee TA (2012) Plasmablastic lymphoma clinically presenting in the urinary tract. Ann Diagn Pathol 16:219-223

89. Touhami H, Brahimi S, Kubisz P, Cronberg S (1987) NonHodgkin's lymphoma of the female urethra. J Urol 137:991-992

90. Truong LD, Caraway N, Ngo T, Laucirica R, Katz R, Ramzy I (2001) Renal lymphoma. The diagnostic and therapeutic roles of fine-needle aspiration. Am J Clin Pathol 115:18-31

91. Tuzel E, Mungan MU, Yorukoglu K, Basakci A, Kirkali Z (2003) Primary renal lymphoma of mucosa-associated lymphoid tissue, Urology 61:463

92. Urban BA, Fishman EK (2000) Renal lymphoma: CT patterns with emphasis on helical CT. Radiographics 20:197-212

93. van den Bosch I, Kropman RF, Blok P, Wijermans PW (2002) Disappearance of a mucosa-associated lymphoid tissue (MALT) lymphoma of the urinary bladder after treatment for Helicobacter pylori. Eur I Haematol 68:187-188

94. Vapnek JM, Turzan CW (1992) Primary malignant lymphoma of the female urethra: report of a case and review of the literature. J Urol 147:701-703

95. Vogeli T, Engstfeld EJ (1992) Non-Hodgkin lymphoma of the female urethra. Scand J Urol Nephrol 26:111-112

96. Wagner JR, Honig SC, Siroky MB (1993) Non-Hodgkin's lymphoma can mimic renal adenocarcinoma with inferior vena caval involvement. Urology 42:720-723 (discussion 723-724)

97. Weimar G, Culp DA, Loening S, Narayana A (1981) Urogenital involvement by malignant lymphomas. J Urol 125:230-231

98. Wick MR, Mills SE, Scheithauer BW, Cooper PH, Davitz MA, Parkinson K (1986) Reassessment of malignant „angioendotheliomatosis". Evidence in favor of its reclassification as ,intravascular lymphomatosis“. Am J Surg Pathol 10:112-123
99. Wronski S, Marszalek A (2011) Diagnostic pitfalls of rare urinary bladder tumors: differential diagnosis of lymphoma-like carcinoma of the bladder-a clinicopathologic study and literature review. J Clin Oncol 29(8):e196-e199. https://doi.org/10.1200/ JCO.2010.32.3121

100. Yang C, Motteram R, Sandeman TF (1982) Extramedullary plasmacytoma of the bladder: a case report and review of literature. Cancer 50:146-149

101. Yasunaga Y, Hoshida Y, Hashimoto M, Miki T, Okuyama A, Aozasa K (1997) Malignant lymphoma of the kidney. J Surg Oncol 64:207-211

102. Zukerberg LR, Harris NL, Young RH (1991) Carcinomas of the urinary bladder simulating malignant lymphoma. A report of five cases. Am J Surg Pathol 15:569-576 